\title{
Adaptive Rate-Dependent Feedforward Controller for Hysteretic Piezoelectric Actuator
}

\author{
U. X. Tan, F. Widjaja, W. T. Latt, K. C. Veluvolu, C. Y. Shee, C. N. Riviere, and W. T. Ang
}

\begin{abstract}
With the increasing popularity of actuators involving smart materials like piezoelectric, control of such materials becomes important. The existence of the inherent hysteretic behavior hinders the tracking accuracy of the actuators. To make matters worse, the hysteretic behavior changes with rate. One of the suggested ways is to have a feedforward controller to linearize the relationship between the input and output. Thus, the hysteretic behavior of the actuator must be first modeled by sensing the relationship between the input voltage and output displacement. Unfortunately, the hysteretic behavior is dependent on individual actuator and also environmental conditions like temperature. In this fast moving world, time is money and it is very costly to model the hysteresis regularly. In addition, the hysteretic behavior of the actuators also changes with age. Base on the studies done on the phenomena hysteretic behavior with rate, this paper proposes an adaptive rate-dependent feedforward controller with Prandtl-Ishlinskii (PI) hysteresis operators for piezoelectric actuators. This adaptive controller is achieved by adapting the coefficients to manipulate the weights of the play operators. Actual experiments are conducted to demonstrate the effectiveness of the adaptive controller.
\end{abstract}

\section{INTRODUCTION}

$\mathrm{H}$ YSTERESIS is a common phenomenon in applications involving issues like magnetic fields and smart materials. One common example of smart materials is piezoelectric actuators. Piezoelectric actuators are playing an ever increasing role in positioning technology. Their applications include cell manipulation, scanning tunneling microscopy and diamond turning machines. One common example of piezoelectric ceramic is PZT ceramic. PZT is a solid solution of $\mathrm{PbZrO}_{3}$ and $\mathrm{PbTiO}_{3}$ and the general formula is $\mathrm{Pb}\left(\mathrm{Zr}_{\mathrm{y}} \mathrm{Ti}_{1-\mathrm{y}}\right) \mathrm{O}_{3}$. PZT has the pervoskite $\mathrm{ABO}_{3}$ structure (Fig. 1).

When a voltage is applied across the ceramic, the atom at the centre ( $\mathrm{Zr}$ or Ti) will displace (Fig. 2). A pole is induced and the net polarization in the PZT ceramic changes. This results in the deformation of the material. The usage of deformation as actuators is a solution for precise actuation as no mechanical backlash is involved. A reverse observation

Manuscript received September 14, 2007. This work was supported in part by College of Engineering, Nanyang Technological University and also by Agency for Science, Technology and Research (A*STAR) SERC grant.

U-Xuan Tan*, Ferdinan Widjaja, Win Tun Latt, Kalyana C. Veluvolu, Cheng Yap Shee, and Wei Tech Ang are with School of Mechanical and Aerospace Engineering, Nanyang Technological University, Singapore. Email: \{ tanu0002, ferd0003, wintunlatt, veluvolu, cyshee, wtang \} @ ntu.edu.sg *Corresponding author

Cameron N. Riviere is with the Robotics Institute, Carnegie Mellon University, Pittsburgh, PA 15213 USA. Email: camr@ri.cmu.edu will occur when the ceramic is loaded. This is why piezoelectric materials are able to function as both actuators and sensors.

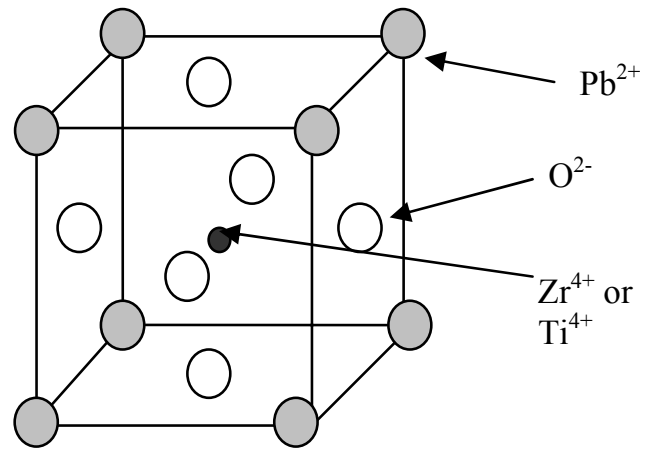

Fig. 1. A Crystal Unit Cell of PZT Ceramic

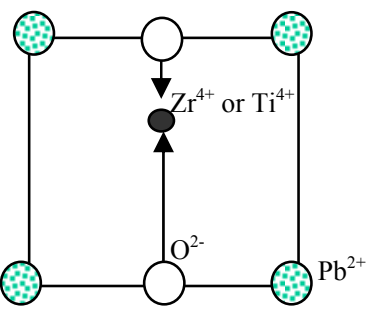

Fig. 2. Polarization.of a PZT Ceramic

However, due to the nature of polarization, such materials exhibits non-linear multi-path hysteresis that makes control challenging, especially if precise tracking is required.

Mathematical hysteresis models are commonly mathematically defined as rate-independent as velocity is not one of the inputs. However, the phenomena hysteretic behavior of most smart materials changes with rate as mentioned in Landauer et al. [1] and Smith et al. [2]. This is due to the nature of the polarization. Thus, a rate-dependent hysteresis model is used.

There are a few proposed methods of controllers to control piezoelectric actuators. One popular method is the usage of a phenomena inverse feedforward controller. As the studies on the hysteretic behavior of such materials are not that well studied at the atom level yet, phenomenological modeling of the hysteretic behavior is commonly used. The inverse model is then used as a feedforward controller to predict and linearize the relationship between the input and output (Fig. 3).

Song et al. [3] proposed using a feedforward model in their controller to improve the performance of the control 
system. Choi et al. [4] also demonstrated that the performance of their PID controller was improved with a feedback linearization loop.

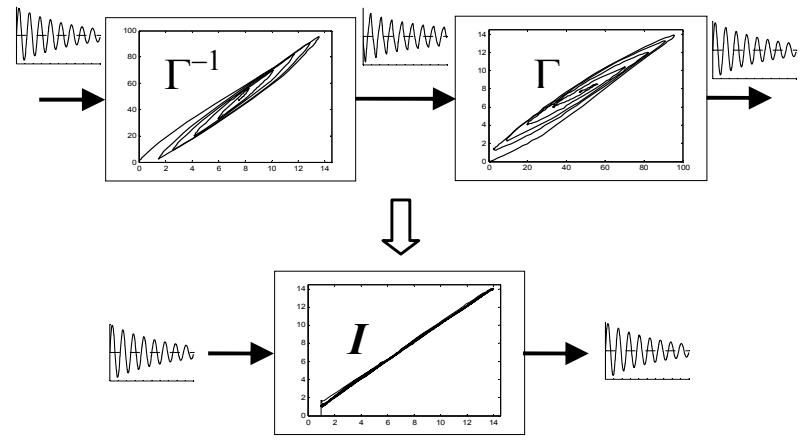

Fig. 3. Linerization of Hysteretic Plant using Inverse Feedforward Controller

There are several mathematical models to model the phenomena hysteretic behavior. Among the simplest way utilize two polynomials. Two sets of polynomial are used to model the forward and backward paths. This method will not work when the turning point is changed as it is not continuous. Sun et al. [5] proposed a new mathematical model by modifying the polynomials.

Among other mathematical hysteresis models, Preisach model [6-8] is the more commonly used model. Maxwell slip model [9] and hysteron model [10] are some other options available.

Prandtl-Ishlinskii (PI) hysteresis model is used in this paper. A major advantage of PI operators is the availability of an analytical inverse model. This is ideal for real-time situation. The inverse model is then used as the feedforward controller to linearize the hysteretic behavior.

The hysteretic behavior of the piezoelectric actuators changes with environment conditions like temperature. In this fast moving world, time is money and it is costly to regularly model each actuator before usage. In addition, the hysteretic behaviors of these actuators change as the actuators age. A couple of adaptive controllers have been proposed [10-11]. However, they are unable to track nonperiodic motions.

Thus, this paper proposed an adaptive rate-dependent hysteresis feedforward controller for piezoelectric actuators. The adaptive controller will account for the dynamic environmental conditions. In addition, this adaptive controller introduces no phase difference and is suitable for tracking purpose.

A brief introduction of the rate-dependent phenomena Prandtl-Ishlinskii hysteresis model is given in the following section. A description of the adaptive controller is given in section III, followed by results, discussions and conclusion.

\section{Hysteresis MATHEMATICAL MODEL}

This section briefly describes the rate-dependent PrandtlIshlinskii hysteresis model. Supplementary details of the
Prandtl-Ishlinskii hysteresis operators can be found in Kuhnen et al. [12-14] and Ang et al. [15].

\section{A. Prandtl-Ishlinskii (PI) Play Operator}

The play operator in the PI hysteresis model, commonly used to model the backlash between gears, is defined by

$$
\begin{aligned}
y(t) & =H_{r}\left[x, y_{0}\right](t) \\
& =\max \{x(t)-r, \min [x(t)+r, y(t-T)]\}
\end{aligned}
$$

where $x$ is the control input, $y$ is the actuator response, $r$ is the control input threshold value or the magnitude of the backlash, and $T$ is the sampling period.

Initial Condition of (1) is given by

$$
y(0)=\max \left\{x(0)-r, \min \left[x(0)+r, y_{0}\right]\right\}
$$

where $y_{0}$ is a real number which is usually initialized to 0 . To change the gradient, a weight value $w_{h}$ is multiplied to the PI operator $H_{r}$. By summing a number of such operators with different threshold values and weights, a hysteresis model is obtained:

$$
y(t)=\vec{w}_{h}^{T} \vec{H}_{r}\left[x, \vec{y}_{0}\right](t)
$$

where weight vector $\vec{w}_{h}^{T}=\left[\begin{array}{lll}w_{h 0} & \ldots & w_{h n}\end{array}\right]$ and $\vec{H}_{r}\left[x, \vec{y}_{0}\right](t)=$ $\left[H_{r 0}\left[x, y_{00}\right](t) \ldots H_{r n}\left[x, y_{0 n}\right](t)\right]^{T}$ with the threshold vector $\vec{r}=\left[\begin{array}{lll}r_{0} & \ldots & r_{n}\end{array}\right]^{T}$ where $0=r_{0}<\ldots<r_{n}$, and the initial state vector $\vec{y}_{0}=\left[\begin{array}{lll}y_{00} & \ldots & y_{0 n}\end{array}\right]^{T}$.

Unlike the Preisach model, which behaves like a flight of stairs, the PI operator is a better mathematical model as it is a first order gradient and it is also more mathematically simpler. To account for the one sided characteristics of piezoelectric actuators, Ang et al. [15] proposed setting the value of $r_{n}$ to be half of the maximum control input.

\section{B. Modified Prandtl-Ishlinskii}

The nature of PI operator is symmetrical about the center point of the loop, but this is not really true in the phenomena observation. To overcome this restriction, Ang et al. [15] and Kuhnen et al. [12] proposed using a saturation operator:

$$
\begin{aligned}
& S_{d}[y](t)= \begin{cases}\max \{y(t)-d, 0\}, & d>0 \\
y(t), & d=0\end{cases} \\
& z(t)=\vec{w}_{s}^{T} \vec{S}_{d}[y](t)
\end{aligned}
$$

The general idea of this saturation operator is to bend the graph and make it not symmetrical. In this paper, the weights of the saturation operator are kept as a constant and will not change in the adapting mechanism.

\section{Rate-Dependent Phenomenological Model}

The phenomena response of the piezoelectric actuator is dependent on the velocity. Thus, experiments are carried out piezoelectric actuators to obtain a rate-dependent phenomena model. The actuator is subjected to different saw-tooth (for constant rate) rate and the hysteresis weights of the play operators are plotted against the control input velocity rate. Experiments are carried out and the result is plotted in Fig. 4. Up to a certain velocity (this limit depends on the length of the piezoelectric ceramic), weights of the play operators of the piezoelectric actuator can be model 
linearly to the velocity input with good approximation.

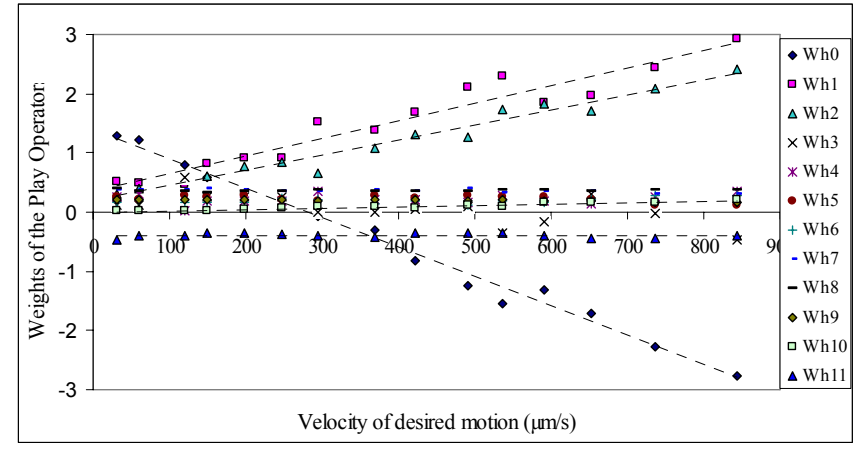

Fig. 4. Weights of the hysteresis play operators against velocity obtained from piezoelectric actuator P885.50 from Physik Instrumente

Thus, base on the phenomena hysteretic behavior with rate, the equation to approximately relate the weights with rate is presented as follows:

$$
w_{h i}(\dot{z}(t))=a_{i}+b_{i} \dot{z}(t), i=0 \ldots n
$$

where $b_{i}$ is the slope of the best-fit line through the $a_{i}$ 's and the referenced slope $a_{i}$ is the intercept of the best-fit line.

Thus the rate-dependent PI model becomes:

$$
z(t)=\Gamma[x, \dot{z}](t)=\vec{w}_{s}^{T} \vec{S}_{d}\left[\vec{w}_{h}^{T}(\dot{z}) \vec{H}_{r}\left[x, \vec{y}_{0}\right]\right](t)
$$

\section{Inverse Model}

Kuhnen [12] showed that the inverse PI model can be expressed by PI play operators too. Thus, the inverse of the rate-dependent PI model can also be expressed as:

$$
\Gamma^{-1}[z](t)=\vec{w}_{h}^{\prime T}(\dot{z}) \vec{H}_{r^{\prime}}\left|\vec{w}_{s}^{T} \vec{S}_{d^{\prime}}[z], \vec{y}_{0}^{\prime}\right|(t)
$$

The inverse of the hysteresis model is basically a reflection along the $45^{\circ}$ line. The inverse model parameters can be calculated by:

$$
\begin{aligned}
& w_{h 0}^{\prime}=\frac{1}{w_{h 0}} ; w_{h i}^{\prime}=\frac{-w_{h i}}{\left(\sum_{j=0}^{i} w_{h j}\right)\left(\sum_{j=0}^{i-1} w_{h j}\right)}, i=1 \ldots n \\
& r_{i}^{\prime}=\sum_{j=0}^{i} w_{h j}\left(r_{i}-r_{j}\right) ; y_{0 i}^{\prime}=\sum_{j=0}^{i} w_{h j} y_{0 i}+\sum_{j=i+1}^{n} w_{h j} y_{0 j} ; \\
& r_{0}=0 ; i=0 \ldots n ; \\
& w_{s 0}^{\prime}=\frac{1}{w_{s 0}} ; w_{s i}^{\prime}=\frac{-w_{s i}}{\left(\sum_{j=0}^{i} w_{s j}\right)\left(\sum_{j=0}^{i-1} w_{s j}\right)}, i=1 \ldots m ; \\
& d_{i}^{\prime}=\sum_{j=0}^{i} w_{s j}\left(d_{i}-d_{j}\right), i=0 \ldots m
\end{aligned}
$$

\section{ADAPTIVE FEEDFORWARD CONTROLLER}

The adaptive feedforward controller is described in this section. The adaptive feedforward controller is first given. The adapting mechanism, which is recursive least square, is next implemented with a slight modification. This section ends with an algorithm of the adapting mechanism for ease in implementation.

\section{A. Adaptive Inverse Hysteretic Controller}

Based on the phenomena hysteretic behavior in section II, it is safe to assume that the hysteretic behavior of a piezoelectric actuator can modeled well using (6). Substituting (5) into (6),

$$
\left.z(t)=\vec{w}_{s}^{T} \vec{S}_{d} \mid \vec{a} \cdot \vec{H}_{r}\left[x, \vec{y}_{0}\right]+\dot{z}(t) \vec{b} \cdot . \vec{H}_{r}\left[x, \vec{y}_{0}\right]\right](t)
$$

Thus, given the desired displacement, the required voltage can be obtained via:

$$
\begin{aligned}
& y_{d}(t)=\vec{w}_{s}^{\prime T} \vec{S}_{d},\left[z_{d}(t)\right] \\
& x(t)=\vec{w}_{h}^{T}(\dot{z}(t)) \vec{H}_{r^{\prime}}\left[y_{d}(t), \vec{y}_{0}^{\prime}\right](t)
\end{aligned}
$$

where $z_{d}(t)$ is the desired displacement, $y_{d}(t)$ is the desired displacement after passing through the inverse saturation operator and $x(t)$ is the estimated required voltage.

Given the input voltage, the actual output displacement from the hysteretic actuator is given as:

$z_{a}(t)=\vec{w}_{s}^{T} \vec{S}_{d}\left[\vec{w}_{h_{p}}^{T}(\dot{z}) \vec{H}_{r}\left[x, \vec{y}_{0}\right] \mid(t)\right.$

where $z_{a}(t)$ is the actual displacement, $\vec{w}_{h_{p}}^{T}$ are the actual weights of the play operators. The actual displacement of the actuator is also the measured displacement. Equation (12) can also be rewritten as:

$$
\begin{aligned}
y_{a}(t) & =\vec{w}_{s}^{T^{T}} \vec{S}_{d^{\prime}}\left[z_{a}(t)\right] \\
& =\vec{w}_{h_{p}}^{T}(\dot{z}) \vec{H}_{r}\left[x(t), \vec{y}_{0}\right] \\
& =\vec{a}_{p} . \vec{H}_{r}\left[x, \vec{y}_{0}\right]-\dot{z}(t) \vec{b}_{p} . . \vec{H}_{r}\left[x, \vec{y}_{0}\right]
\end{aligned}
$$

The error of the model is obtained as:

$$
\begin{aligned}
e_{m}(t) & =y_{a}(t)-y_{d}(t) \\
& =\vec{w}_{s}^{T} \vec{S}_{d^{\prime}}\left[z_{a}(t)\right]-\vec{w}_{h}^{T}(\dot{z}(t)) \vec{H}_{r}\left[x(t), \vec{y}_{0}\right] \\
& =\vec{w}_{s}^{T} \vec{S}_{d^{\prime}}\left[z_{a}(t)\right]-\vec{a} \cdot \vec{H}_{r}\left[x, \vec{y}_{0}\right]-\dot{z}(t) \vec{b} . \vec{H}_{r}\left[x, \vec{y}_{0}\right]
\end{aligned}
$$

In this paper, to adapt the vectors $\vec{a}$ and $\vec{b}$, least square is performed with the cost function:

$V(t)=\frac{1}{2} \sum_{i=0}^{t}\left[e_{m}(i)\right]^{2}$

Assuming the hysteretic behavior of a piezoelectric actuator can be modeled accurately using equation (6), the vectors $\vec{a}$ and $\vec{b}$ will adapt as close to the behavior of the plant as possible. In other words,

$\lim _{t \rightarrow \infty} e_{m}(t) \approx 0$

$\lim _{t \rightarrow \infty} a_{i} \approx a_{i_{a}}$ and $\lim _{t \rightarrow \infty} b_{i} \approx b_{i_{a}}$ for $i=1, \ldots, n$

With the adapted vectors $\vec{a}$ and $\vec{b}$ that can closely model the plant, $\vec{w}_{h}^{T}(\dot{z})$ can be obtained. Thus, the inverse $\vec{w}_{h}^{T}(\dot{z})$ can also be obtained using equation (8) and the desiredactual error will be minimized. The signal flow diagram of the adaptive feedforward controller is given in Fig. 5. 


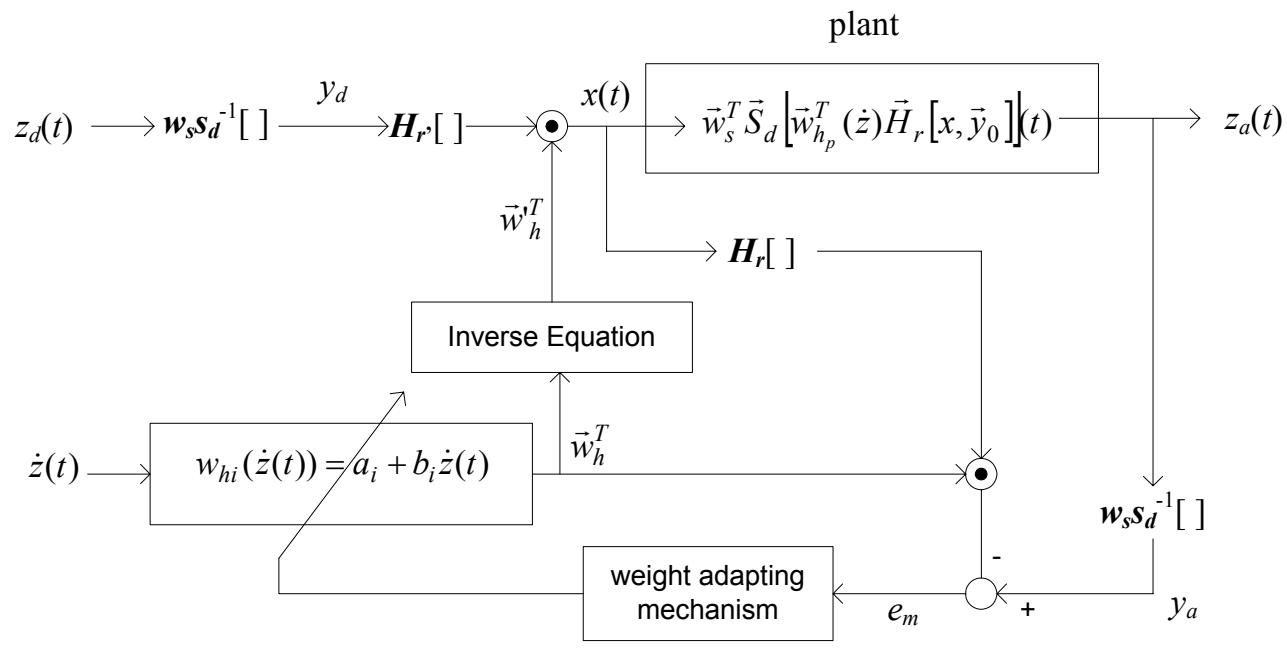

Fig. 5. Signal Flow Diagram of the Adaptive Feedforward Controller

\section{B. Adaptive Weight-Control Mechanism}

The input vector to the recursive least square (RLS) is:

$\vec{I}(t)=.\left[H_{r_{0}}, \dot{z}(t) \cdot H_{r_{0}}, H_{r_{1}}, \dot{z}(t) \cdot H_{r_{1}}, \ldots H_{r_{n}}, \dot{z}(t) \cdot H_{r_{n}}\right]^{T}$

where $H_{r_{i}}$ is $H_{r_{i}}\left[x, y_{0}\right]$. It is more important to adapt the $a_{i}$

values to the hysteretic behavior than $b_{i}$. It can also be seen in Fig. 4 that the gradient of the first few weights are more significant. Thus, the terms in the input vector, $\vec{I}(t)$, is multiplied with the terms in a priority vector, $\left[c_{a 0}, c_{b 0}, \ldots\right.$ $\left.c_{a n}, c_{b n}\right]^{T}$, to obtain:

$\mathbf{u}(t)=\left[c_{a 0} H_{r_{0}}, c_{b 0} \dot{z}(t) \cdot H_{r_{0}}, \ldots, c_{a n} H_{r_{n}}, c_{b n} \dot{z}(t) \cdot H_{r_{n}}\right]^{T}$

In this paper, $c_{a i}$ is set to 1 and $c_{b i}$ is set to 0.0008 if the unit of $\dot{z}(t)$ is in $\mu \mathrm{m} / \mathrm{s}$.

The weight vector to be adapted is defined as:

$\hat{\mathbf{w}}(t)=\left[a_{0}, b_{0}, a_{1}, b_{1}, \ldots, a_{n}, b_{n}\right]^{T}$

The remaining derivation steps to obtain the algorithm are very similar to the derivation of adaptive filters using RLS and can be found in [15]. Other available adaptive laws found in adaptive control textbooks may be implemented, with some modification if needed.

\section{Algorithm}

Initialization of the weights is first performed as shown in (21). Note that the first weight must again be set to be a positive non-zero number. The matrix $\mathbf{P}$ is also initialized. After that, for each sample loop, the parameters are computed as described in (22).

Initialization:

$\hat{\mathbf{w}}(0)=(5,0 \ldots)^{\mathrm{T}}$

$\mathbf{P}(0)=\delta^{-1} \mathbf{I}$

where $\delta$ is a positive real number called the regularization parameter and is set to be a small positive constant for high signal to noise ratio (SNR) and set to be a large positive constant for low SNR.
For each instant of time, $n=1,2, \ldots$, use

$\boldsymbol{\pi}(n)=\mathbf{P}(n-1) \mathbf{u}(n)$,

$\boldsymbol{\mu}(n)=\boldsymbol{\pi}(n) /\left\{\lambda+\mathbf{u}^{H}(n) \boldsymbol{\pi}(n)\right.$,

$\hat{\mathbf{w}}(n+1)=\hat{\mathbf{w}}^{\prime}(n)+\boldsymbol{\mu}(n) \mathbf{u}(n) e_{m}(n)$,

$\mathbf{P}(n)=\lambda^{-1} \mathbf{P}(n-1)-\lambda^{-1} \boldsymbol{\mu}(n) \mathbf{u}^{H}(n) \mathbf{P}(n-1)$.

where $\lambda$ is the forgetting factor which is a positive constant close to, but less than 1 . When $\lambda$ is set to 1 , it implies infinite memory. Thus, set $\lambda$ to be near unity if you want a controller that will remember most of the historical result, but it means it will adapt slower to a changing plant.

\section{EXPERIMENTAL RESULTS}

In this section, a brief description of the experiment setup is first given, followed by the results. The behavior of the piezoelectric actuator P885.90 from Physik Instrumente is used to demonstrate the adaptive rate-dependent feedforward controller.

\section{A. Experiment Setup}

As seen from Fig. 6, a 16-bit D/A card is used to generate the necessary voltage, which is then passed through the amplifier (the gain is approximately 10). Given the voltage, the actuator will deform and the interferometer will detect the displacement and convert it to analog voltage signal. Using a 16-bit $\mathrm{A} / \mathrm{D}$ card, the $\mathrm{PC}$ reads in the displacement.

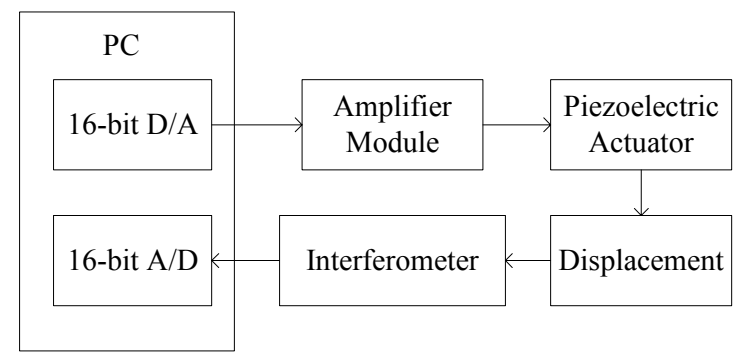

Fig. 6. Experimental Architecture

To perform tracking control, real-time operating system 
QNX Neutrino is used. The sampling rate of the DAQ card is set at $10 \mathrm{kHz}$. A timer interrupt is set at an interval of 0.5 ms $(2 \mathrm{kHz})$ to average the data collected and update the parameters. The averaging acts a form of filtering.

\section{B. Experimental Results}

Experiments are conducted to demonstrate the performance of the adaptive feedforward controller. The objective of the actuator is to actuate according to a desired motion. In all the experiments conducted for this paper, the parameters of the actuator are treated as unknown. The first term of vector $\mathbf{a}$ is initialized as 5 whilst all the remaining terms in the vectors $\mathbf{a}$ and $\mathbf{b}$ are all initialized as 0 .

The first experiment is to actuate the actuator in a periodic sinusoidal motion. The rmse is found to be $0.1211 \mu \mathrm{m}$ between the time of $0.75 \mathrm{~s}$ to $1 \mathrm{~s}$. The rmse will continue to drop if more adaptation time is permitted. The rmse from time $10 \mathrm{~s}$ to $20 \mathrm{~s}$ is $0.0584 \mu \mathrm{m}$, which is near to the interferometer's noise level. Using a sensor with lower noise and better resolution might be able to reduce the rmse.

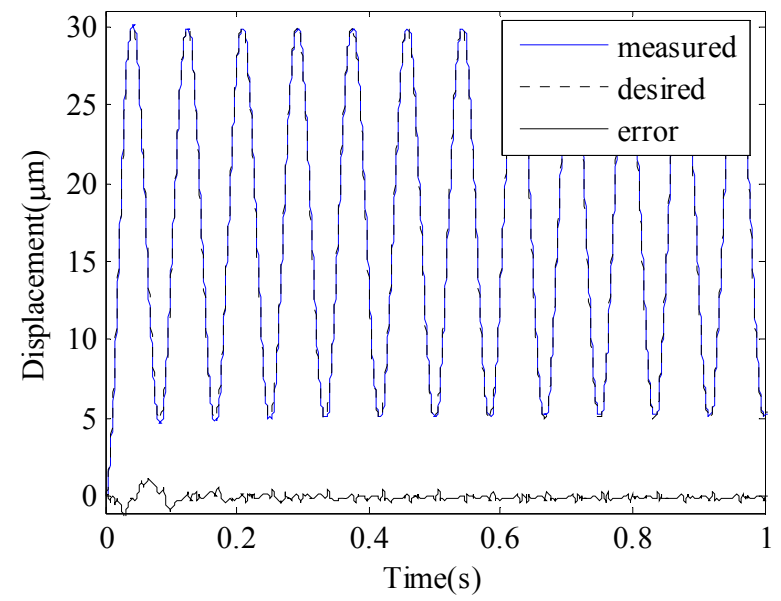

Fig. 7. Tracking result of a $12 \mathrm{~Hz}$ Sinusoidal wave.

TABLE I

Measured Performance of THE AdAPTIVE RATE-DEPENDENT CONTROLLER IN TRACKING SINUSOIDAL WAVEFORMS.

\begin{tabular}{lccc}
\hline \hline & $4 \mathrm{~Hz}$ & $8 \mathrm{~Hz}$ & $12 \mathrm{~Hz}$ \\
\hline $\begin{array}{l}\text { rmse }(\mu \mathrm{m}) \\
\begin{array}{l}\text { rmse / actuator's } \\
\text { stroke length }\end{array}\end{array}$ & 0.0638 & 0.0619 & 0.0584 \\
\hline
\end{tabular}

The rmse and max errors are the results taken from over a span from 10s to 20 s.

In the first experiment, the desired motion is a periodic motion. However, the desired motions in many industrial applications are random and non-periodic. Thus, the adaptive feedforward controller was tested with a number of randomly created desired waveform motions. These waveforms are created by random values. These values are then passed through a low pass filter to smoothen out the points. Random numbers are used to ensure that the waveform is not periodic. This is where the rate-dependent phenomena play a role in the adaptive feedforward controller.

Five different waves were randomly created. Fig. 8 shows how the weights converge while Fig. 9 shows the tracking performance of the adaptive feedforward controller for one of the waveforms. A section between time $5 \mathrm{~s}$ and $8 \mathrm{~s}$ is shown in Fig. 10. The result is summarized in Table II. A wide time span of 10 s to 20 s is used to calculate the result for better representation. The mean rmse of the 5 nonperiodic motions is found to be $0.0943 \mu \mathrm{m}$ with $\sigma=0.0159$. The mean of the maximum errors is $0.3899 \mu \mathrm{m}$ with $\sigma=$ 0.0291 .

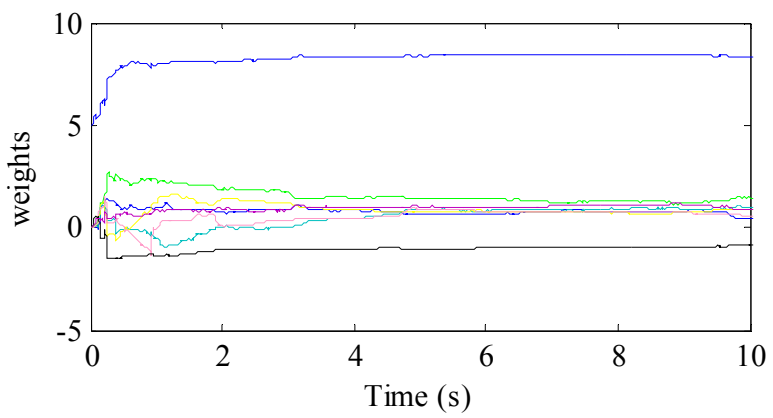

Fig. 8. Convergence of the weights of the adapting mechanism.

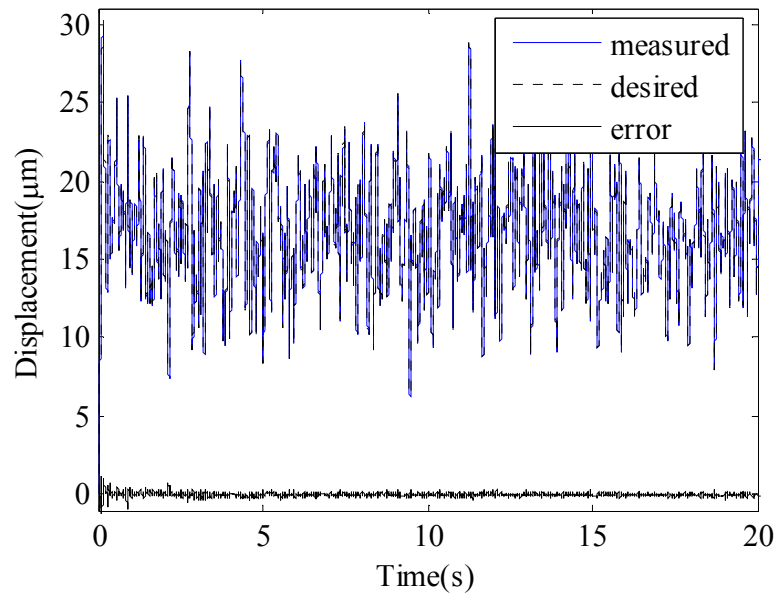

Fig. 9. Experimental results of a random non-periodic wave. The different non-periodic waves are created by passing random numbers through a low pass filter.

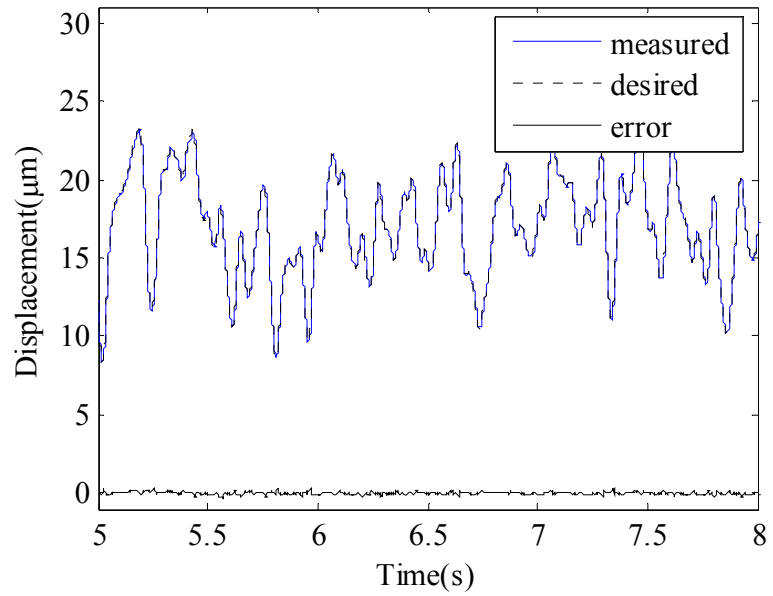

Fig. 10. A Section of Fig. 9. between time $5 \mathrm{~s}$ to $8 \mathrm{~s}$. 
TABLE II

MEASURED PERFORMANCE OF THE ADAPTIVE RATE-DEPENDENT CONTROLLER IN TRACKING RANDOMLY CREATED WAVEFORMS.

Adaptive Rate-dependent Controller

\begin{tabular}{lc}
\hline rmse $\pm \sigma(\mu \mathrm{m})$ & $0.0943 \pm 0.0159$ \\
rmse / peak-peak $(\%)$ & 0.33 \\
max error $\pm \sigma(\mu \mathrm{m})$ & $0.3899 \pm 0.0291$ \\
max error / peak-peak $(\%)$ & 1.35 \\
\hline
\end{tabular}

The rmse and max errors are the mean results over a set of 5 different random non-periodic waveform experiments. The error is calculated from time $=10 \mathrm{~s}$ to $20 \mathrm{~s}$. The waveforms are created by passing random numbers through a low-pass filter. The peak-peak is obtained as the difference between the maximum and minimum during this 10 s interval of the random non-periodic waveforms.

\section{DISCUSSION}

Most controllers like PID controllers introduce phase difference. Phase difference comes with higher tracking error. The proposed adaptive hysteresis feedforward controller is suitable for tracking purpose as no phase difference is introduced.

In this paper, the actuator is actuated in accordance to the non-periodic random waveform. Thus, there is no prior knowledge of the desired motion and the motion is nonperiodic. The purpose of choosing non-periodic waveform is to illustrate the rate-dependent ability of the adaptive feedforward control. As illustrated in the experiments, although the hysteretic plant (actuator) is treated as unknown, the adaptation speed of the feedforward controller is less than a second. The convergence speed is dependent on the waveform. A good learning waveform at the beginning will shorten the convergence time. Different adapting mechanism/law can be used too. In this paper, RLS is used as it is a simple and good algorithm with fast convergence time to implement. The author had also implemented least mean square (LMS) as the adapting mechanism. Although LMS is more robust to sudden changes, the steady state error is not as low and also the environmental conditions do not often change abruptly.

However, there are two main limitations. The input signals must satisfy the persistent excitation condition. In addition, to obtain the inverse parameters, it is assumed that the gradient at all the points are positive. In other words, the sum of weights at any point is assumed to be positive. Negative gradients must be avoided at all times. The authors plan to modify [17] and implement in this controller.

\section{CONCLUSION}

The hysteretic behavior of a piezoelectric actuator changes with environment conditions and age. Thus, an adaptive rate-dependent hysteresis feedforward controller for hysteretic plants is proposed and presented. This is achieved by adapting the coefficients that relate rate to the weights of the play operators to suit the environment. Actual implementation of the adaptive controller was carried out on a piezoelectric actuator. The rate-dependent property of the adaptive feedforward controller was illustrated with the tracking control ability of non-periodic random waveforms which most controllers are unable to perform.

\section{ACKNOWLEDGMENT}

The author would like to thank Dr. Klaus Kuhnen from University of Saarland, Germany, for clearing the author's doubt on some issues.

\section{REFERENCES}

[1] R. Landauer, D. R. Young, and M. E. Drougard, "Polarization reversa in the barium titanatehysteresis loop," Jouranl of Applied Physics, vol. 27(71), pp. 752-758, 1956.

[2] R. C. Smith, Z. Ounaies, and R Wieman, "A Model for Ratedependent Hysteresis in Piezoceramix Materials Operating at Low Frequencies", Technical Report, NASNCR-2001-211-62, NASA Langley Research Center, 2001.

[3] G. Song, J. Zhao, X. Zhou, and J. A. D. Abreu-Garcia, "Tracking control of a Piezoceramic Actuator with Hysteresis Compensation using inverse Preisach Method," IEEE/ASME Tracnsactions on Mechatronics, vol. 10, April 2005.

[4] G. S. Choi, H. S. Kim, and G. H. Choi, "A Study on Position Control of Piezoelectric Actuators," Int. Symposium on Industrial Electronics, Guimaraes, Portugal, 1997.

[5] L. Sun, C. Ru, W. Rong, L. Chen, and M. Kong, "Tracking Control of Piezoelectric Acuator based on a new mathematical model," Jouranal of Micromechanics and Microengineering, vol. 14, no. 11, pg 1439. 1444, 2004

[6] H. Hu and R. B. Mrad, "On the Classical Preisach model for hysteresis in piezocramic actuators," Mechatron, vol. 13, pp. 85-92, 2002.

[7] D. Hughes and J. T. Wen, "Preisach Modeling of Piezoceramic and Shape Memory Alloy Hysteresis," 4th IEEE Conf. on Control Applications, 1995.

[8] X. Tan and J. S. Batas,"Modeling and control of hysteresis nmagnetostrictive actuators," Automatica, pp. 1469-1480, 2004.

[9] M. Goldfarb, and N. Celanovic, "A lumped parameter electromechanical model for describing the nonlinear behavior of piezoelectric actuators," Journal of Dynamics, Systems, Measurements and Control, vol. 119, no. 3, pg 478-485, 1997.

[10] G. Tao, "Adaptive Control of Plants with Unknown Hystereses," IEEE Transcaions on Automatic Control, 1995.

[11] Y. Feng, Y. X. We, Y. M. Hu, and C. Y. Su, , "Adaptive backstepping control of a class of uncertain nonlinear systems with PrandtlIshlinskii hysteresis", $4^{\text {th }}$ International Conference on Machine Learning and Cybernetics, pp 697-01, Guangzhou, China, Aug., 2005.

[12] K. Kuhnen, "Modeling , Identification and Compensation of Complex Hysteretic Nonlinearities: A modified Prandtl-Ishlinskii Approach," European Journal of Control, Vol. 9, No. 4, pp. 407-418, 2003.

[13] K. Kuhnen and H. Janocha, "Inverse Feedforward Controller for Complex Hysteretic Nonlinearities in Smart-Material Systems,' Control and Intelligent System, vol. 29, pp. 74-83, 2001.

[14] P. Krejci, and K. Kuhnen, "Inverse Control of System with Hysteresis and Creep," IEE Proc.of Control Theory and Applications, vol. 148, no. 3, pp. 185-192, May, 2001.

[15] W. T. Ang, C. N. Riviere, and P. K. Khosla "Feedforward Controller with Inverse Rate-Dependent Model for Piezoelectric Actuators in Trajectory Tracking Applications," ASME/IEEE Tansactions on Mechatronics, Vol. 12, No. 2, pp. 1-8, April, 2007.

[16] S. Haykin, Adaptive Filter Theory, $3^{\text {rd }}$ Edition, Prentice Hall, New Jersey, 1996.

[17] U. X. Tan, T. L. Win, and W. T. Ang, "Modeling Piezoelectric Actuator Hysteresis with Singularity Free Prandtl-Ishlinskii Model,' IEEE Int. Conf. on Robotics \& Biomimetics, Kunming, China, 2006. 